\title{
Alter
}

Revue de phénoménologie

$25 \mid 2017$

L'Histoire

\section{Heidegger et le problème de l'accès phénoménologique à la réalité historique}

François Jaran

\section{CpenEdition}

\section{Journals}

Édition électronique

URL : http://journals.openedition.org/alter/450

DOI : 10.4000/alter.450

ISSN : 2558-7927

Éditeur :

Association ALTER, Archives Husserl (CNRS-UMR 8547)

\section{Édition imprimée}

Date de publication : 3 décembre 2017

Pagination : $59-77$

ISBN : 978-2-9550449-3-3

ISSN : $1249-8947$

\section{Référence électronique}

François Jaran, «Heidegger et le problème de l'accès phénoménologique à la réalité historique », Alter [En ligne], 25 | 2017, mis en ligne le 01 décembre 2018, consulté le 21 avril 2019. URL : http:// journals.openedition.org/alter/450 ; DOI : 10.4000/alter.450 


\section{HEIDEGGER ET LE PROBLÈME DE L'ACCÈS PHÉNOMÉNOLOGIQUE À LA RÉALITÉ HISTORIQUE}

François Jaran

L'histoire et l'historicité constituent deux éléments d'une importance inestimable dans la construction de la phénoménologie heideggérienne. Le caractère historique de l'existence humaine, d'une part, représente incontestablement le point d'achoppement central de sa reprise de la méthode husserlienne et définit la teneur de cette phénoménologie aux accents herméneutiques ${ }^{1}$. D'un autre côté, l'histoire pensée comme région ontologique et comme thème $d^{\prime}$ une science possible - cette question si sensible des débats entre Dilthey et les néokantiens - a aussi préoccupé le philosophe sur le chemin qui le mène à la rédaction de Sein und Zeit.

Nous chercherons, dans les pages qui suivent, à montrer comment la phénoménologie qu'élabore Heidegger au tout début des années vingt s'intéresse à la donation phénoménologique de l'histoire à partir d'une analyse de ses diverses manifestations. Dans ces textes finalement peu commentés, Heidegger montre que dans certains des rapports qu'établit l'existence humaine avec lui, l'historique acquiert une existence propre. Le problème ainsi posé établit une distance importante avec la question simplement épistémologique que posaient Dilthey et les néokantiens et prend la forme d'une ontologie phénoménologique de l'histoire. ${ }^{1}$ Nous avons consacré un ouvrage à la question: Phénoménologies de l'histoire. Husserl, Heidegger
et l'histoire de la philosophie, Louvain, Peeters, 2013. 


\section{Le problème d'une ontologie phénoménologique de l'histoire}

Si nous jetons un bref coup d'œil au projet d'une " ontologie fondamentale » présenté dans Sein und Zeit, il apparaît immédiatement que l'histoire y joue un rôle fondamental. Le squelette de cette œuvre inachevée le montre parfaitement : la première partie - partiellement publiée en 1927 - présente une structure systématique qui cherche à offrir la "réponse concrète » à la question de l'être ${ }^{2}$ en mettant en évidence le caractère temporel (zeitlich) de notre compréhension de l'être et le caractère temporal (temporal) de l'être ; la seconde partie qui n'existe pas comme telle mais qui devait constituer la seconde moitié de l'ouvrage - était censée poursuivre un but essentiellement historique, c'est-à-dire qu'elle aurait dû consister en une «destruction de l'histoire de l'ontologie au fil conducteur de la problématique de la temporalité (Temporalität) $»^{3}$.

Mais l'importance de l'histoire dans Sein und Zeit ne se limite pas à cette seconde partie inexistante. Heidegger parle aussi de l'histoire dans l'œuvre publiée, et ce à deux moments importants. Dans l'introduction $(\S 6)$, alors qu'il présente la structure du traité, il insiste sur l'importance méthodologique de la destruction et sur la structure nécessairement historico-systématique de toute réflexion philosophique. Comme Heidegger l'écrit alors, la philosophie ne peut se réaliser, ne peut être complète, si elle ne reconquiert pas tout d'abord « la transparence de sa propre histoire $»^{4}$. Plus loin, Heidegger reviendra sur le thème de l'histoire lorsqu'il élabore sa réflexion sur la relation entre la temporalité du Dasein et son historicité (cinquième chapitre de la seconde section, § 72-77).

Le 31 décembre 1927, quelques mois après la publication de Sein und Zeit, Heidegger tente de dresser un portrait de sa propre philosophie pour un article d'encyclopédie que son ami Rudolf Bultmann doit rédiger. Dans cette brève tentative pour systématiser sa contribution philosophique, Heidegger insiste d'une façon tout à fait surprenante sur le rôle que joue l'histoire dans sa pensée. Lisons les indications que donne Heidegger à Bultmann :

\footnotetext{
${ }^{2}$ M. Heidegger, Sein und Zeit (dorénavant SZ), Tübingen, Niemeyer, 18e éd., 2001, p. 19 (Être et Temps, trad. E. Martineau, Paris, Autentica, 1985). Nous n'indiquons la pagination des traductions françaises - que nous avons toujours consultées quitte à les modifier - que lorsque la pagination de l'original allemand n'est pas reprise en marge.

${ }_{3}^{3}$ SZ, p. 39.

${ }_{4} \mathrm{SZ}, \mathrm{p} .22$.
} 
Heidegger et le problème de l'accès phénoménologique à la réalité...

Je trouve un peu étrange l'article « $\mathrm{H}$ [eidegger] » alors que je commence à peine à marcher à quatre pattes. On ne peut aborder la chose autrement que par une énumération des motifs qui, pour ainsi dire, me composent. En ce qui concerne le contenu, il faudrait simplement dire que mon travail tend vers une radicalisation de l'ontologie antique et, simultanément, vers un élargissement (Ausbau) universel de celle-ci en rapport avec la région de l'histoire (die Region der Geschichte) $)^{5}$.

Dans cette lettre, Heidegger reconnaît que son travail - c'est-à-dire cette tâche qu'il a entreprise avec Sein und Zeit et sur laquelle il travaille encore à cette date précise $-s$ 'articule autour de deux tâches essentielles.

Dans un premier temps, " il tend vers une radicalisation de l'ontologie antique ». Dans les cours qui précèdent la publication de Sein und Zeit ainsi que dans l'œuvre elle-même, Heidegger a souligné en quel sens son projet ontologique devait être considéré comme plus radical que celui des Grecs. Cela se justifie de deux façons : tout d'abord, les Grecs n'ont pas distingué l'être et l'étant et n'ont donc pas pu poser adéquatement la question de l'être formulée dans Sein und Zeit. Ensuite, les Grecs n'ont pas explicitement découvert que la question devait se poser depuis un étant privilégié, soit depuis le Dasein.

Dans un second temps - ou plutôt : «simultanément » comme l'écrit Heidegger à Bultmann -, le projet d'une ontologie fondamentale tend « vers un élargissement universel de [l'ontologie antique] en rapport avec la région de l'histoire ». Ainsi, Heidegger ne prétend pas uniquement radicaliser le questionnement ontologique sur l'être, mais aussi l'élargir, en cherchant une façon de le mettre en rapport avec ce qu'il appelle « la région de l'histoire ».

Cette description de la seconde tâche de l'ontologie fondamentale peut s'interpréter de deux manières. Tout d'abord, en radicalisant l'ontologie antique de telle sorte qu'elle prenne conscience de son "fundamentum ontique » que représente le Dasein, Heidegger se voit obligé d'intégrer l'histoire à l'ontologie dans la mesure où le Dasein est un étant qui se caractérise par son historicité et où la compréhension de l'être qui lui appartient est elle aussi déterminée par une certaine histoire. C'est la manière même d'interroger l'être à partir de la compréhension historique de l'être qui oblige Heidegger à tenir

\footnotetext{
${ }^{5}$ R. Bultmann et M. Heidegger, Briefwechsel 1925 bis 1975, A. Grossmann et C. Landmesser (éd.), Francfort-sur-le-Main, Klostermann, 2009, p. 47. Il existe une traduction de cette lettre due à Claudia Serban et Laurent Villevieille publiée dans Archives de sciences sociales des religions, ${ }^{\circ}$ 171, 2015/3, p. 243.
} 
compte de l'histoire. De fait, l'histoire dans Sein und Zeit, c'est-à-dire l'historicité, apparaît surtout comme un des éléments rendant possible le questionnement au sujet de l'existence humaine et, conséquemment, celui au sujet de l'être.

Mais l'affirmation de Heidegger permet aussi une autre interprétation. Il évoque en effet un «élargissement universel » de l'ontologie «en rapport avec la région de l'histoire». La seconde interprétation serait la suivante: il ne s'agit pas seulement de reconnaître l'importance qu'a l'histoire en tant que condition de possibilité de la réflexion ontologique, mais d'abord et surtout d'élargir le "champ d'application" de l'ontologie en direction de l'histoire. Ainsi, l'ontologie n'aurait pas seulement pour «champ d'application » les étants qui se tiennent là-devant (vorhanden), les étants naturels, la région «nature »comme cela a toujours été le cas depuis les Grecs -, mais elle pourrait aussi discourir sur l'essence et l'existence des étants historiques, de la région « histoire». De cette façon, l'ontologie fondamentale tenterait une chose que très peu de penseurs ont osé tout au long de l'histoire : poser la question du mode d'être des étants historiques ou, en d'autre mots, se demander de quelle façon existent les réalités dont la particularité est d'être historiques.

Si cette question doit se convertir en une interrogation proprement ontologique, c'est-à-dire en une question portant sur l'être de l'étant, il faut alors que nous soyons à même d'identifier des étants qui présentent un tel mode d'être. Une telle approche exige une redéfinition $\mathrm{du}$ " caractère historique » de telle sorte que celui-ci ne soit pas réduit à un attribut propre à ce qui a cessé d'exister et soit plutôt repensé comme un trait de l'étant qui fait encontre dans le monde, de l'étant intramondain.

On sait que dans Sein und Zeit et dans les textes de la même époque, c'est le Dasein qui est dit être «primairement historique » (primär geschichtlich) ${ }^{6}$. Mais si le Dasein l'est avant tout autre étant, c'est parce qu'il y a des étants qui le sont de façon dérivée, comme c'est le cas des « antiquités » étudiées dans l'œuvre de 1927. Selon ces lignes du $\S 76$ de Sein und Zeit, les antiquités sont des étants qui font encontre dans le monde mais qui se caractérisent par leur appartenance au monde d'un Dasein « ayant-été-là » (dagezesenes Dasein $)^{7}$. Ce sont des objets qui apparaissent bel et bien dans notre monde et qui, pourtant, manifestent une référence évidente à un monde qui n'est plus.

${ }^{6} \mathrm{SZ}, \mathrm{p} .381$.

7 SZ, p. 393-394. 
Heidegger et le problème de l'accès phénoménologique à la réalité...

Certains textes de l'époque attestent cependant que Heidegger était loin d'avoir offert par là une réponse achevée aux problèmes d'une ontologie de $l^{\prime}$ histoire ${ }^{8}$. Si l'on se fie à la caractérisation qu'il fait de son travail dans la lettre à Bultmann de décembre 1927, on peut penser qu'il s'agissait alors d'une tâche à laquelle Heidegger comptait s'atteler dans les sections inédites de Sein und Zeit ou dans un autre contexte. Mais comme nous le verrons ici, c'est aussi une question à laquelle Heidegger avait déjà fourni une certaine réponse dans ses premiers cours de Fribourg, une réponse qui va au-delà de la simple analyse phénoménologique de l'antiquité présentée en 1927.

Ce chapitre de Sein und Zeit consacré à l'historicité et à la temporalité (§ 72-77), on le sait, a été principalement écrit sous l'influence

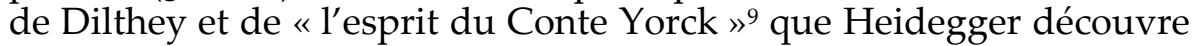
en 1923 lorsqu'est publiée la correspondance entre les deux amis. Mais avant de découvrir cet « esprit » qui défend avec intelligence la " différence générique » entre " $\mathrm{l}^{\prime}$ ontique et $\mathrm{l}^{\prime}$ historique ${ }^{10}-\mathrm{c}^{\prime}$ est-àdire, dans la terminologie de Yorck, entre ce qui est purement matériel et ce qui, comme l'existence humaine, doit être compris à partir de l'historicité -, Heidegger avait déjà saisi que l'histoire est un phénomène qui doit être étudié depuis la perspective de la facticité de l'existence humaine et non à partir des particularités méthodologiques des sciences de l'esprit, comme le défendaient ses maîtres néokantiens. Afin de bien comprendre la position de Heidegger au sujet de "l'historique », nous commencerons par présenter ce qu'il écrit dans le cours de l'hiver 1920-1921 intitulé Einleitung in die Phänomenologie der Religion. Nous nous pencherons ensuite sur l'analyse du phénomène de l'histoire que Heidegger livre en 1920 dans son cours Phänomenologie der Anschauung und des Ausdrucks.

\section{Penser l'historique depuis l'expérience facticielle de la vie}

Dans ces cours qui sont antérieurs à la «découverte » qu'il fera bientôt de la fonction fondamentale et fondatrice de la question de l'être, la préoccupation principale de Heidegger est ce qu'il appelle la

\footnotetext{
${ }^{8}$ Voir, entre autres, M. Heidegger, Die Grundprobleme der Phänomenologie, GA 24, p. 240-241 (Les problèmes fondamentaux de la phénoménologie, trad. J.-F. Courtine, Paris, Gallimard, 1985) et Einleitung in die Philosophie, GA 27, p. 393-394.

${ }_{9}$ SZ, p. 404.

${ }_{10}$ Briefwechsel zwischen Wilhelm Dilthey und dem Grafen Paul Yorck v. Wartenburg, 1877-1897, E. Rothacker (éd.), Halle-sur-Saale, Max Niemeyer Verlag, 1923, p. 191.
} 
vie facticielle ${ }^{11}$. Il considère alors que le problème central de la philosophie est celui de la vie humaine pensée non pas comme un objet général tel qu'un anthropologue pourrait l'étudier, mais bien comme un objet particulier pensé depuis sa facticité. Ce que Heidegger prétend appréhender dans cette étude, c'est l'existence humaine dans son déploiement même, pour la saisir non pas comme une généralité mais bien comme une chose qui est en train de m'arriver à moi-même en cet instant. La vie facticielle, soutient Heidegger, n'est pas une chose qui puisse être captée comme un mécanisme réitérable tel la digestion ou la respiration. La vie facticielle est à chaque fois mienne et ne peut être pensée autrement que comme mienne dans la mesure où il est exclu que je fasse facticiellement l'expérience d'autres existences.

Les premiers textes que Heidegger consacre au problème de «l'historique » portent encore la marque de son aversion juvénile pour l'histoire ${ }^{12}$ dans la mesure où, contrairement aux textes plus tardifs, ceux-ci cherchent encore à légitimer le caractère proprement historique de la philosophie et critiquent la thèse selon laquelle l'histoire constituerait plutôt une menace pour la philosophie. Depuis Platon, la philosophie aurait cherché «l'éternellement valide» et a donc souhaité se maintenir éloignée de ce qui est historique, compris comme ce qui change constamment, comme le contingent par excellence $^{13}$. Heidegger cherche précisément à défendre le contraire : en réalité, «la problématique philosophique tire principiellement sa motivation de ce qui est historique ${ }^{14}{ }^{14} c^{\prime}$ est-à-dire que la philosophie ne peut jamais échapper à l'historique puisqu'elle en émerge. Heidegger soutient alors que la philosophie ne pourra jamais s'élaborer indépendamment de l'histoire et donc que la distinction entre la philosophie systématique et la philosophie historique est sans fondement. Pour lui, on ne peut travailler à résoudre des «problèmes

\footnotetext{
11 À la suite de Jean-François Courtine et de Jean Greisch, nous avons choisi de traduire faktisch par « facticiel», plutôt que par « factif ».

12 Voir M. Heidegger, «Lebenslauf (Zur Habilitation 1915)», Reden und andere Zeugnisse eines Lebensweg, Gesamtasugabe (dorénavant GA), tome 16, Francfort-sur-le-Main, Klostermann, 19752017 , p. 39. Pour ce qui est du rôle que jouera le professeur Heinrich Finke dans sa « conversion " à l'histoire, on consultera l'intéressant texte de Gabriel Cercel, "Sur la valeur du comprendre historique pour le philosophe" : Martin Heidegger et Heinrich Finke ", Bulletin Heideggérien I, dans Archives de Philosophie, n 74, 2011, p. 365-372.

${ }_{13}$ M. Heidegger, Phänomenologie der Anschauung und der Ausdruck. Theorie der philosophischen Begriffsbildung, GA 59, p. 43 (Phénoménologie de l'intuition et de l'expression. Théorie de la formation des concepts philosophiques, trad. G. Fagniez, Paris, Gallimard, 2014) et M. Heidegger, Phänomenologie des religiösen Lebens, GA 60, p. 31 (Phénoménologie de la vie religieuse, trad. J. Greisch, Paris, Gallimard, 2012).

${ }_{14}$ GA 60, p. 31.
} 
Heidegger et le problème de l'accès phénoménologique à la réalité...

systématiques » sans connaître l'histoire de ces mêmes problèmes ni connaître l'histoire d'un problème sans s'en occuper philosophiquement ${ }^{15}$. Cette conviction est bien sûr une constante dans toute l'œuvre de Heidegger et dans la tradition herméneutique qui suivra. Le philosophe doit être historien et l'historien de la philosophie doit être philosophe ${ }^{16}$.

Mais que signifie que la philosophie soit un «phénomène historique » (historisches Phänomen)? Heidegger souligne que cela ne peut pas simplement indiquer que la philosophie - comme c'est aussi le cas des courses de voitures et de l'évaporation de l'eau - se déroulerait dans le temps. La philosophie n'est pas située dans le temps de la même façon que les autres choses le sont. Pour comprendre le caractère historique de la philosophie, il est nécessaire de souligner, comme le fait Heidegger, que celle-ci a partie liée avec l'existence facticielle.

Cela est affirmé dans une phrase dont la traduction est problématique : «Savoir de quelle manière est possible une telle caractérisation de la philosophie [sc. une caractérisation qui en fait un phénomène historique] est problématique ; en tout cas, elle besteht dans l'expérience facticielle de la vie $»^{17}$. On aura compris, le verbe bestehen est celui qui pose problème dans la mesure où, lorsqu'il est accompagné de la préposition in, il signifie généralement " consister dans/en ». Dans la phrase qui nous intéresse, une telle traduction nous inviterait à penser que Heidegger considère que pour résoudre le problème de la caractérisation historique de la philosophie, il faut commencer par saisir que cette dernière consiste dans l'expérience facticielle de la vie ce qui ne peut pas être le cas ici puisque l'expérience facticielle de la vie n'est pas la philosophie, mais bien son point de départ, son objet ou encore l'endroit où celle-ci se déploie. En ce sens, il nous paraît beaucoup plus cohérent de traduire bestehen par exister ou subsister ${ }^{18}$.

\footnotetext{
${ }^{15}$ M. Heidegger, Zur Bestimmung der Philosophie, GA 56/57, p. 125.

${ }^{16}$ C'était d'ailleurs déjà ce qu'il défendait dans sa thèse d'habilitation (Die Kategorien- und Bedeutungslehre des Duns Scotus in M. Heidegger, Frühe Schriften, GA 1, p. 196-204; Traité des catégories et de la signification chez Duns Scot, trad. F. Gaboriau, Paris, Gallimard, 1970).

${ }_{17}$ GA 60, p. 32.

${ }^{18}$ Jean Greisch choisit de traduire par "réside dans », ce qui maintient d'une certaine façon l'ambiguïté du texte allemand. "La philosophie réside dans l'expérience de la vie » peut en effet signifier qu'elle se déploie en son sein ou encore qu'elle consiste en elle. Les traducteurs espagnols (J. Uscatescu, Introducción a la fenomenología de la religión, Madrid, Siruela, 2005) et italiens (G. Gurisatti, Fenomenologia della vita religiosa, Milan, Adelphi, 2003) optent pour cette seconde traduction qui nous parait insoutenable: que la philosophie consiste dans (consiste en/consiste in) l'expérience facticielle de la vie. Seul le traducteur anglais entend la phrase de la même façon que nous en traduisant bestehen par subsist. Il faut noter que dans les années qui suivent, Heidegger utilisera le verbe bestehen et le substantif Bestand pour caractériser le mode d'être de l'étant qui n'est à la mesure ni du Dasein, ni de l'étant vorhanden, ni de l'étant zuhanden,
} 
Pour des raisons qui s'éclairciront plus loin, il nous paraît important d'insister sur la portée ontologique du verbe bestehen. Ce que Heidegger affirme ici, c'est que le caractère historique de la philosophie peut s'expliquer à partir du fait suivant : elle existe au sein de l'expérience facticielle de la vie. La philosophie, en ce sens, est « contaminée » par le caractère facticiel et historique de l'existence humaine. Cela implique que l'essence de la philosophie ne peut être déterminée à partir d'une réflexion sur l'éternel et sur les façons qu'elle a de nous tenir éloignés de la contingence historique.

Avec cette affirmation, Heidegger prétend montrer que plutôt que d'en faire un objet que l'on aborderait grâce à des concepts généraux, l'historique doit être assimilé à un concept au plus près de l'existence humaine. Afin d'y parvenir, Heidegger analyse l'expérience propre de la vie humaine non pas en termes généraux mais dans son déploiement facticiel. Cette manière d'exposer qui cherche à me parler de la philosophie et de l'histoire au moment même où je suis en train d'exister, et qui repousse les discussions éloignées de la vie immédiate qui s'établissent au niveau purement conceptuel et théorique, est tout à fait propre de cette pensée élaborée au cours des années vingt et jusque dans Sein und Zeit. Heidegger ne souhaite pas établir ce qu'est l'histoire en soi ou mettre la main sur un concept général sous lequel subsumer toutes les expériences historiques. Ce qui l'intéresse est de découvrir ce qu'est l'histoire pour moi à qui elle se manifeste et qui peux la comprendre au sein même de mon expérience facticielle de la vie.

Selon la pré-saisie (Vorgriff) que nous avons de l'histoire, tout objet qui se déroulerait et se modifierait au fil du temps serait "historique » (historisch). Ainsi, les guerres humaines, l'évolution d'une langue, mais aussi tout processus qui demande du temps : une réaction chimique donnée ou le mouvement d'une onde. Selon cette définition on ne peut plus ample de l'histoire, les seules choses qui ne seraient pas historiques seraient celles qui se situent à l'extérieur du temps : l'eau, la diagonale ou tout concept universel dont l'existence n'est qu'idéale. Mais cette définition trop ample ne nous explique aucunement pourquoi la philosophie devrait être considérée comme historique en un sens spécifique. Heidegger remet en doute cette définition et se pose la question de savoir "s'il existe la possibilité de mettre au jour un autre sens du terme "historique" comme tel, et qui

ni de l'animal, ni de la plante et qui, pourtant, n'est pas rien, comme c'est le cas de l'espace et du nombre (GA 27, p. 71 ; voir aussi SZ, p. 7). La philosophie - et l'histoire, nous le verrons plus loin - ont très certainement un mode d'être apparenté à ces réalités. 
Heidegger et le problème de l'accès phénoménologique à la réalité...

ne se laisse pas du tout attribuer en ce sens aux objets (Objekten) ». Et si le concept actuel de l'historique n'était «qu'un dérivé de ce sens originaire $»^{19}$ ? Un concept de l'historique qui ne signifie rien d'autre que «se déroulant dans le temps» ou «temporel» n'a en effet aucune signification philosophique. Lorsque nous cherchons à saisir l'historique, nous avons en tête quelque chose qui permet de distinguer certains étants des autres, puisque de certains étants, nous ne dirons jamais qu'ils sont historiques même s'ils sont situés dans le temps. Comme dans bien d'autres cas, Heidegger affirme qu'il existe des concepts originaires et des concepts dérivés et que, souvent, on prend initialement les premiers pour les seconds. Ici, nous pourrions en effet penser que sont tout d'abord historiques tous les objets situés dans le temps et que ce ne serait que dans un second temps que nous décidons de transformer le sens de l'adjectif «historique » pour l'appliquer uniquement aux étants qui ont une certaine signification historique. Mais Heidegger tend à penser de façon non traditionnelle la relation entre les concepts originaires et dérivés. Selon lui, l'existence humaine comprend tout d'abord qu'il y a au sein de sa facticité des objets qui possèdent un sens historique accentué. Ce n'est que dans un second temps qu'elle en vient à découvrir que pratiquement tous les objets se déroulent dans le temps. Voyons comment Heidegger parvient à défendre cette position.

Pour commencer, Heidegger doit rejeter la démarche d'une «philosophie de l'histoire »- soit la démarche que l'on rencontre dans le Geschichtsphilosophie de Rickert - qui considère la connaissance historique comme le mode d'accès privilégié aux étants historiques. Ce qui intéresse Heidegger, c'est davantage «l'historique tel qu'il nous vient à l'encontre dans la vie» et non «dans la science historique $»^{20}$. L'historique ne se manifeste pas à nous parce que nous sommes des historiens, mais bien parce que nous sommes des "êtres historiques», comme l'écrit Dilthey, ou des "animaux qui ont une histoire », comme l'affirme Windelband ${ }^{21}$. L'histoire est donc une chose que l'on rencontre tout d'abord dans la vie humaine et seulement dans un second temps grâce à la science historique. Le résultat de l'appréhension individualisante de Rickert qui caractérise tout objet individuel comme historique - au sens ample de « qui a lieu dans le

\footnotetext{
${ }^{19}$ GA 60, p. 32.

${ }^{20} \mathrm{Idem}$.

${ }^{21}$ Respectivement, W. Dilthey, Der Aufbau der geschichtlichen Welt in den Geisteswissenschaften, GS VII, p. 278 et W. Windelband, "Geschichte und Naturwissenschaft», in Präludien. Aufsätze und Reden zur Philosophie und ihrer Geschichte, Tübingen, J. C. B. Mohr, 1915, vol. 2, p. 152 ; «Histoire et sciences de la nature », trad. S. Mancini, Les études philosophiques, no 1, 2000, p. 11.
} 
temps » - n'est qu'un trait dérivé d'un sens originaire que Heidegger cherche précisément à trouver. Avant de supporter un tel "vidage scientifique et théorique ", l'historique est tout d'abord un "phénomène vivant », la «spontanéité immédiate de la vie » ${ }^{22}$.

La cible de l'attaque de Heidegger, c'est ici la logique de l'histoire de Rickert qui, selon lui, n'a aucune sensibilité (Fühlung) pour «l'historicité vivante qui est parvenue pour ainsi dire à ronger de l'intérieur notre existence ${ }^{23}$. Heidegger s'intéresse à l'historicité, c'est-àdire au caractère historique de ma propre existence et non au trait méthodologique de l'appréhension scientifique. Le point de départ de la recherche heideggérienne ne sera donc pas l'historicité des choses en général, mais bien l'historicité vivante, c'est-à-dire cette historicité qui « dévore » mon existence.

Les questions que l'on doit se poser sont donc les suivantes: Comment vit-on l'histoire ? Comment se présente l'histoire au sein de notre existence même ? Selon ce qu'écrit Heidegger, l'histoire est bien souvent une " charge ${ }^{24}$ dans la mesure où elle nous poursuit partout où nous allons. Même lorsque nous ne pensons pas directement aux objets historiques, nous adoptons un point de vue distinct sur notre monde par le simple fait de savoir qu'ont existé des gens par le passé et qu'il existe des cultures distinctes de la nôtre, des visions différentes du monde. Tout cela détermine notre expérience de la vie d'une manière déterminante. Si nous parvenions à saisir l'histoire à partir de sa manifestation au sein de notre existence, nous ferions dès lors face au phénomène originaire de l'histoire dont sont dérivés tous les autres, y-compris la science historique.

Cette approche nouvelle, Heidegger l'oppose à tout ce qui se fait en philosophie de l'histoire à son époque, c'est-à-dire non seulement au néokantisme, mais aussi aux travaux d'Oswald Spengler et de Georg Simmel. Dans toutes ces approches, selon Heidegger, on traite l'histoire comme s'il s'agissait d'un objet saisi à partir de la connaissance que l'on en a, depuis l'étude thématique propre à l'attitude théorique. C'est précisément le contraire que Heidegger propose : explorer l'historique depuis la vie même, c'est-à-dire non pas lorsqu'il se constitue en objet d'étude scientifique, mais lorsque nous vivons en lui. En adoptant l'attitude théorique, il est certainement possible de contempler notre sujet d'étude, mais nous le faisons au prix d'une suspension de «la référence vivante à l'objet de connais-

${ }^{22}$ GA 60, p. 33.

${ }^{23}$ Idem.

24 Ibid., p. 38. 
Heidegger et le problème de l'accès phénoménologique à la réalité...

sance $»^{25}$. De cette façon, nous perdons le sens profond de l'historique qui n'est pas une chose que l'on doive étudier en milieu clos, mais qui, en un sens fondamental, nous inquiète. La philosophie, selon Heidegger, n'aurait jamais fait que nous tranquilliser face à cette menace que constitue l'historique.

C'est donc dans l'expérience facticielle que nous en faisons que l'historique doit nous indiquer ce qu'il en est de lui. Mais cette position laisse plusieurs questions en suspens :

Comment est lié l'historique à l'existence facticielle de la vie ? Quel sens présente l'historique au sein de l'existence facticielle de la vie? [...] Qu'entend-on exactement ici par « historique $»^{26}$ ?

\section{Premier essai d'une ontologie phénoménologique de l'histoire}

Dans le texte de 1920-1921 que nous venons de commenter, Heidegger ne donne pas de réponse «concrète » au problème de l'historicité et renvoie plutôt son auditeur à une explication méthodologique sur la formalisation et l'indication formelle ${ }^{27}$. Mais dans le cours antérieur, celui de l'été 1920, Heidegger avait offert beaucoup d'informations au sujet de ce que pourrait être une " phénoménologie de l'histoire » qui ose prendre l'existence facticielle comme point de départ. Pour bien comprendre ce que cela signifie, il faut évoquer même de façon trop succincte - comment fonctionne la phénoménologie heideggérienne. De façon générale, nous pouvons affirmer que pour Heidegger, pratiquer la phénoménologie, c'est tout d'abord interroger en direction de l'essence et de la manière d'être des choses à partir d'une analyse des façons de se comporter (sich benehmen) propres à l'existence humaine et par lesquelles elle établit un rapport (sich beziehen) à ces choses qui se manifestent ${ }^{28}$. Ces comportements du Dasein ont cette particularité qu'ils peuvent être réitérés par chacun de nous dans la mesure où il en a une connaissance intime, les

\footnotetext{
${ }^{25}$ Ibid., p. 48.

${ }^{26}$ Ibid., p. 54.

${ }^{27}$ Ibid., p. 55 sq. Pour ce qui est du rôle de l'indication formelle chez Heidegger, nous renvoyons au troisième chapitre de Ramón Rodríguez, Fenómeno e interpretación. Ensayos de fenomenología hermenéutica, Madrid, Tecnos, 2015.

${ }_{28} \mathrm{Au}$ sujet de ce concept phénoménologique de «comportement » (Verhalten) dont le sens est double, voir M. Heidegger, Phänomenologische Interpretationen zu Aristoteles. Einführung in die phänomenologische Forschung, GA 61, p. 52 sq. (Interprétations phénoménologiques en vue d'Aristote. Introduction au cour de la recherche phénoménologique, trad. P. Arjakovsky et D. Panis, Paris, Gallimard, 2016).
} 
ayant souvent vécus lui-même. Nous reconnaissons ici la méthode que Heidegger emploie aussi à d'autres moments, comme lorsqu'il analyse les diverses façons d'être dans la vérité dans le Natorp Bericht de l'automne 1922 (GA 62), mais aussi lorsqu'il analyse dans Sein und Zeit l'être de l'étant intramondain dont les structures ontologiques sont définies à partir de la teneur (Gehalt) découverte au sein des comportements du Dasein que sont la considération théorique et le comportement pratique. À l'été 1920, Heidegger expose de façon on ne peut plus claire la manière dont le Dasein se fraie un chemin vers l'histoire, c'est-à-dire les diverses modalités dans lesquelles le Dasein rencontre l'histoire. En interrogeant l'histoire, Heidegger adopte ici cette méthode phénoménologique qui lui est propre et formule ses questions de la façon suivante: au sein de l'existence humaine (im menschlischen Dasein), quels comportements permettent à l'histoire de se manifester? Comment se manifeste l'histoire dans chacun des rapports que le Dasein établit?

Ainsi, l'analyse que propose Heidegger s'appuie sur les divers comportements qu'adopte le Dasein et dans lesquels la chose signifiée par le concept d'histoire se donne ou est rendue manifeste. Le premier comportement $\left(n^{\circ}\right.$ I) qu'il analyse, c'est celui que, dans une perspective opposée, nous devrions considérer d'emblée comme le plus essentiel : le comportement associé à la science historique. Heidegger soutient que bien que ces comportements soient loin d'être les plus naturels et les plus courants au sein de l'existence humaine, cela ne signifie pas qu'ils n'ont pas d'existence. Ainsi, lorsque l'on adopte les comportements typiques d'un historien - assister à des conférences ou à des cours, consacrer quelques heures par jour à lire des livres à la bibliothèque, etc. -, «l'histoire» se manifeste comme la « science historique ». Dans ce contexte concret et propre à l'existence humaine, un certain accès à l'histoire s'ouvre, même s'il ne s'agit aucunement de l'accès le plus «naturel».

Ce premier accès à l'histoire nous renvoie à un autre sens (no II) contenu dans l'équivocité essentielle du concept d'histoire. Curieusement, nous donnons aussi le nom d' « histoire » à l'objet qu'étudie la science historique. L'histoire n'est donc pas qu'une science, elle est aussi et sans doute surtout ce que nous appelons les événements historiques. En un premier temps, Heidegger évite la question liée à la teneur concrète de ces événements, mais évoque déjà une distinction importante :

L'histoire, dit-on, signifie quelque chose d'objectif : ce qui a eu lieu (das, was geschehen ist), le passe (die Vergangenheit), ce qui est passé (das Vergangene). On vise par là tout ce qui a jamais eu lieu, en le 
Heidegger et le problème de l'accès phénoménologique à la réalité...

prenant dans la plus large extension. Nous n'examinons pas la question de savoir si tout ce qui a eu lieu (alles Geschehene) est déjà lui-même historique, ou si l'historique (das Historische) ne constitue qu'une coupe au sein de ce qui a eu lieu ${ }^{29}$.

La seconde signification de l'histoire semble donc permettre une subdivision importante, celle qui existe entre l'ensemble le plus ample de tout ce qui est advenu et l'ensemble des événements qui ont "laissé une trace » et que Heidegger caractérise ici comme l'historique. On touche ici à un problème central de la philosophie de l'histoire telle que la déploie à l'époque Rickert. Celui-ci donne le nom $\mathrm{d}^{\prime}$ "universum historique » à "l'ensemble historique le plus vaste possible, donc une chose unique et individuelle selon son concept, à laquelle appartient en tant qu'élément individuel tout objet traité par une étude historique spécifique ${ }^{30}$, l'ensemble sur lequel se découpe l'histoire au sens de ce qui a déjà subi une sélection s'opérant à partir $\mathrm{du}$ filtre que constituent les valeurs.

Heidegger distingue donc comme le faisait avant lui Rickert entre «ce qui est historique » (das Historische) et " ce qui a eu lieu » (das Geschehene). Mais ce type de distinction, remarque Heidegger, provient d'une attitude bien particulière, soit l'attitude théorique. Si nous nous maintenons dans le cadre phénoménologique heideggérien, nous pouvons nous demander: au sein de quel type de comportement s'accomplit la manifestation de "ce qui est historique " et de «ce qui a eu lieu»?

Dans la mesure où il s'agit de concepts abstraits, idéaux qui jamais ne se manifestent dans notre vie, nous pourrions penser qu'ils n'entretiennent aucune relation avec les comportements humains. L'histoire n'aurait de réalité ici qu'indépendamment de l'existence humaine et on pourrait penser que la phénoménologie heideggérienne devrait refuser de les considérer comme d'authentiques phénomènes historiques. Mais Heidegger apporte d'importantes nuances : il est vrai que ni l'advenu ni l'historique ne se manifestent dans la vie humaine quotidienne puisqu'ils ne sont que de pures abstractions. Néanmoins, on les découvre dans un certain type de comportement : les comportements théoriques et scientifiques qui pensent le passé comme étant détaché de l'existence humaine. En tant qu'objet de la pensée, le «passé objectif » maintient un rapport fort à un comporte-

\footnotetext{
${ }^{29}$ GA 59, p. 50.

${ }^{30}$ H. Rickert, Die Probleme der Geschichtsphilosophie. Eine Einführung, Heidelberg, Carl Winter, 1924, p. 98 ; Les problèmes de la philosophie de l'histoire. Une introduction, trad. mod. B. Hébert, Toulouse, Presses Universitaires du Mirail, 1998, p. 130.
} 
ment humain concret. Heidegger écrit : «On aura tôt fait, sans doute, de signaler que cette totalité objective d'être n'est rien si elle n'est pas le corrélat d'une appréhension sinon actuelle, du moins possible ${ }^{31}$. Le passé objectif se donne donc comme une "idée purement spéculative " au sein d'un comportement possible, et ce qui se manifeste dans ce comportement comme histoire doit bel et bien être considéré comme une façon qu'a l'histoire de se rendre manifeste: ou bien comme l'idée d'un ensemble de tout ce qui a eu lieu (« universum historique ») ou bien comme l'idée de l'ensemble réduit des événements que l'on considère à bon droit comme historiques (historisch).

Ainsi, les trois premiers sens que prend l'histoire (comme science, comme l'ensemble de ce qui a eu lieu, comme l'historique) se manifestent au sein d'un comportement bien précis qui se caractérise à partir de son indépendance par rapport à la réalité humaine. Heidegger souligne en effet que " l'histoire comme cette totalité objective d'être n'est pas nécessairement rapportée à une existence (Dasein) actuelle déterminée $»^{32}$. L'histoire comme concept scientifique et objectif, au même titre que la nature, a cette prétention d'exister indépendamment de la vie facticielle qui l'appréhende: "Le passé objectif : ce qui a eu lieu, pensé comme détaché de tout rapport d'appréhension historique déterminé, en dehors d'un présent historique déterminé $»^{33}$. Mais dans ces trois cas, le passé est précisément "pensé comme» (gedacht als). Le concept d'histoire vise donc ici nécessairement le corrélat d'un être-pensé. Heidegger remarque en effet que cette "détermination théorétique [...] pose initialement une idée, en l'occurrence celle de l'histoire comme celle d'un être passé (ein vergangenes Sein) ${ }^{34}$. Mais cette idée est, selon Heidegger, le résultat d'une "détermination idéalisante sur le mode théorétique qui fait abstraction de tout présent déterminé ${ }^{35}$, c'est-à-dire qui fait abstraction du présent depuis lequel ce passé est appréhendé. C'est la perspective qu'adopterait un sujet parfaitement idéal s'il pouvait jeter un regard sur le passé. Il s'agit d'un rapport d'appréhension objectivant qui prétend se détacher complètement du présent qui existe concrètement (konkrete daseiende Gegenwart). L'histoire pensée comme " passé objectif » est donc une "idée purement spéculative», une relation conçue idéalement depuis un sujet idéal. Nous voyons ici clairement que nous sommes très loin de cette conception de l'histo-

\footnotetext{
31 GA 59, p. 64.

32 Ibid., p. 64-65.

${ }^{33}$ Ibid., p. 65.

${ }^{34}$ Idem.

${ }^{35}$ Idem.
} 
Heidegger et le problème de l'accès phénoménologique à la réalité...

rique que Heidegger présentait comme rongeant l'existence humaine depuis l'intérieur.

Mais au-delà des considérations théoriques sur l'histoire, Heidegger soutient que l'histoire se donne aussi de façon bien distincte dans d'autres comportements humains, c'est-à-dire lorsque la vie humaine ou les communautés humaines "entrent en rapport» avec le passé d'autres vies ou d'autres communautés. Heidegger donne divers exemples des façons dont s'établissent ces rapports ( $\mathrm{n}^{\circ}$ III, IV et V).

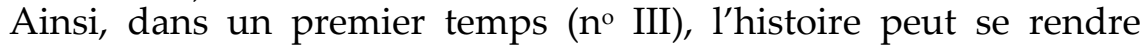
manifeste lorsqu'un peuple, une collectivité, une tribu, un groupe humain entre en rapport avec son propre passé, grâce aux préceptes de ses ancêtres, aux lois héritées, aux fables racontées, aux symboles propres, etc., ce qu'en un mot nous appelons la tradition. Selon ce qu'écrit Heidegger, cette appropriation de la tradition peut se faire de façon explicite ou non, mais la présence d'historiens professionnels $\mathrm{n}^{\prime}$ est pas pertinente puisque dans ces cas, ce n'est jamais la science historique qui établit les liens entre générations.

Dans un second temps ( $\left.n^{\circ} \mathrm{IV}\right)$, Heidegger commente le proverbe selon lequel l'histoire serait «la maîtresse de la vie » (Historia vitae magistra). Cela signifie fondamentalement que nous rencontrons l'histoire dans les modèles et les leçons qu'elle peut nous donner et qui orientent notre vie. Ici, nous pourrions donner des exemples concrets d'hommes d'état qui ont cherché à ne pas répéter des erreurs historiques, mais Heidegger pense le proverbe en un sens beaucoup plus ample, comme une caractéristique de la vie humaine qui constamment maintient un rapport avec ce qui a eu lieu dans les vies d'autrui. Au sein de notre propre vie, de façon constante et sans que nous ayons à nous arrêter pour lire des livres d'histoire, nous comptons toujours déjà sur l'histoire. L'être humain maintient un rapport de familiarité avec le passé des existences d'autrui et oriente de façon implicite ses actions sur ce qu'il sait des existences passées.

Mais l'histoire se manifeste aussi à un niveau encore plus individuel $\left(n^{\circ} \mathrm{V}\right)$ lorsque $j^{\prime}$ entre en rapport avec ma propre histoire, lorsque je contemple les événements qui ont eu lieu au cours de ma vie. Je me souviens, par exemple, avoir vécu dans une autre ville à un autre moment de mon existence ou avoir exercé un autre métier.

De cette façon, Heidegger montre que l'histoire se manifeste dans ces rapports "naturels" qui sont autant de manières $\mathrm{d}^{\prime}$ " avoir » l'histoire : une collectivité «a » son histoire, un individu «a » l'histoire d'autrui ou sa propre histoire. Nous reconnaissons ici l'influence de Dilthey qui expliquait les mécanismes grâce auxquels la vie entre 
en rapport avec elle-même ou en rapport avec les vies d'autrui. Mais ce que nous souhaitons souligner ici, c'est surtout la «hiérarchie » qu'il nous est loisible de déduire de ce que Heidegger affirme.

Une fois établi l'ensemble des sens que peut prendre le mot « histoire ${ }^{36}$, Heidegger insiste sur le fait que les sens III à V sont les sens les plus authentiques de l'histoire. Le critère que Heidegger utilise ici est celui de la relation avec l'existence humaine, c'est-à-dire avec l'existence facticielle telle qu'il l'entend alors. Les deux premiers sens sont d'emblée exclus: le premier (la science historique), parce qu'elle est un mode de comportement qui sans nul doute se donne dans l'existence facticielle mais qui est loin d'être une relation normale et courante avec l'histoire ; le second (l'ensemble de ce qui a eu lieu et l'historique), parce qu'il ne constitue qu'une «idée purement spéculative », un rapport conçu idéalement depuis un sujet idéal et non depuis l'expérience facticielle de la vie.

Ces deux premiers sens contrastent donc avec les autres concepts d'histoire dans lesquels celle-ci apparaît dans un véritable rapport de l'existence humaine avec le passé. Heidegger n'hésite d'ailleurs pas à considérer ces rapports comme "authentiques" (genuin) face aux autres rapports. De quelle façon sont-ils davantage « authentiques »? Heidegger écrit qu'au sein de ces rapports établis avec l'existence humaine concrète et contrairement aux deux premiers sens, ce à quoi on se réfère avec le mot histoire « existe facticiellement ». Dans ces cas où elle est pensée comme un rapport entre la vie facticielle et les événements passés, l'histoire ne se donne pas comme un concept purement abstrait, mais comme quelque chose de facticiel :

Contrairement [aux sens I et II], le rapport authentique [établi] en III, IV et V a ceci de caractéristique que ce qui est visé par "histoire» n'obtient qu'en lui et par lui son sens concret, c'est-à-dire que le rapport reconduit de son côté à un Dasein concret et qu'en ce Dasein, grâce au rapport, ce qui est visé par «histoire » existe facticiellement $^{37}$.

\footnotetext{
${ }^{36}$ Nous laissons volontairement de côté le dernier sens ( ${ }^{\circ}$ VI) auquel Heidegger donne finalement peu d'importance et qui se réfère aux expressions du type « il m'est arrivé une drôle d'histoire».

37 « Dagegen hat in III, IV und V der genuine Bezug das Eigentümliche, daß in ihm und durch ihn das mit Geschichte Gemeinte erst seinen konkreten Sinn erhält, d. h. daß der Bezug seinerseits auf ein konkretes Dasein zurückweist und in diesem durch den Bezug faktisch das besteht, was mit Geschichte gemeint ist »(GA 59, p.65-66). Notre traduction diffère grandement de celle de Guillaume Fagniez qui donne un autre sens à la problématique expression bestehen in (qui signifie «consister dans » ou " exister au sein de ») : « c'est-à-dire que le rapport reconduit de son côté à une existence concrète, et que ce qui est visé par "histoire" consiste, à travers ce rapport factif, en cette existence même» (p. 87 de la traduction). Notons tout d'abord que faktisch ne peut ici être traduit comme un adjectif (facticiel ou factif) se rapportant au mot « rapport» mais prend bien
} 
Comme l'écrit Heidegger, dans les rapports III, IV et V, l'histoire est véritablement «tenue au sein de l'accomplissement» (gehabt im Vollzug) $)^{38}$, contrairement aux premières formes qui ne constituent pas un tenir ou un avoir l'histoire mais un simple connaître l'histoire. Les premières considérations, explique Heidegger, présupposent toujours l'élimination (Zerstörung) de l'existence facticielle. L'être humain et son existence concrète, individuelle et historique sont niés au sein de ces conceptions de l'histoire auxquelles il est fait référence dans les deux premiers sens, c'est-à-dire lorsque l'histoire est pensée comme science ou comme passé objectif.

Si ces analyses de Heidegger peuvent être mises au compte d'une " ontologie de l'histoire », alors la question du mode d'être des étants historiques reçoit ici une première réponse. L'histoire est certes

plutôt la forme d'un adverbe qui ne peut que déterminer le verbe bestehen. Ce que l'on appelle « histoire» doit donc "consister en quelque chose » ou " exister dans quelque chose » de façon facticielle. La première raison qui nous incline à traduire bestehen par « exister » est que les seuls textes de la GA où Heidegger emploie ce verbe avec l'adverbe faktisch font référence à l'existence de fait, à l'existence facticielle d'une chose - une science, une question, une variété (respectivement: Grundprobleme der Phänomenologie, GA 58, p. 241 ; Platon: Sophistes, GA 19, p. 467 ; Aristoteles, Metaphysik $\theta$ 1-3. Von Wesen und Wirklichkeit des Kraft, GA 33, p. 200) - et jamais au fait qu'une chose consiste facticiellement en une autre. Grammaticalement, il semblerait néanmoins plus prudent de traduire comme le fait Fagniez et d'interpréter la phrase comme si Heidegger cherchait à affirmer que "l'histoire consiste en ce Dasein », c'est-à-dire que l'histoire et l'existence humaine fonctionnent comme des équivalents, ce qui, tout comme c'était le cas pour la philosophie dans l'autre texte dont la traduction a été commentée plus haut, est tout simplement inexact. Lorsque l'histoire devient manifeste au sein d'une existence facticielle selon les modalités expliquées dans les rapports III, IV et V, on ne peut aucunement affirmer que celle-ci devrait alors être considérée comme "consistant en l'existence ». Il nous apparaît donc beaucoup plus près du sens du texte et de l'emploi que fait généralement Heidegger du verbe bestehen au sens d' " exister " (voir, par exemple, GA 59, p. 20, 32, 57 et 86) d'interpréter le texte de la façon suivante : " l'histoire existe facticiellement dans l'existence humaine lorsqu'elle est appréhendée dans ces trois rapports concrets ». Pour cela, il faut aussi comprendre le « in diesem [Dasein] » au sens de «dans le Dasein », c'est-à-dire "au sein de l'existence » - ce qui est une façon tout à fait usuelle qu'a Heidegger d'employer l'expression, par exemple lorsqu'il affirme que la philosophie et l'histoire sont des guises existant au sein même de l'existence («...sind es im Dasein seiende Weisen », Ontologie. Hermeneutik der Faktizität, GA 63, p. 48-49; Ontologie. Herméneutique de la factivité, trad. A. Boutot, Paris, Gallimard, 2012). Dans le cas de cette traduction du verbe bestehen, notre choix coïncide avec celui que font Tracy Colony ( i.e. that the relation for its part points back to a concrete Dasein and that, in the latter, by virtue of the relation, what is meant by history factically exists ", Phenomenology of Intuition and Expression. Theory of Philosophical Concept Formation, Londres, Continuum, 2010) et Armando Canzonieri (" poiché il rapporto rimanda in se stesso a un esserci concreto e per il fatto che, in questo esserci, in virtù del rapporto, ciò che è inteso con storia esiste in maniera effettiva ", Fenomenologia dell'intuizione e dell'espressione. Teoria della formazione del concetto filosofico, Macerata, Quodlibet, 2012).

38 GA 59, p. 62. 
présente de diverses façons, mais jamais aussi authentiquement que lorsqu'elle se manifeste dans la vie facticielle elle-même, lorsque le Dasein ouvre un accès à l'histoire d'autrui et à la sienne propre. C'est dans de tels comportements que se révèle primairement ce que nous appelons l'histoire et vient à l'existence comprise comme Bestand. En un sens secondaire et dérivé, nous pouvons aussi considérer que la science historique ainsi que le passé objectif existent, mais ce ne sera alors que de façon «idéale » et au sein de comportements théoriques qui ne sont certes pas inauthentiques mais qui ne sont ni courants ni originaires. La hiérarchie ontologique s'établit ici de façon on ne peut plus claire : l'histoire n'existe véritablement que lorsque je la considère depuis ma propre existence facticielle et dans la mesure où elle se manifeste au sein de celle-ci.

Heidegger restera fidèle à cette façon de penser l'histoire à partir de sa manifestation originaire dans le Dasein jusqu'à Sein und Zeit au moins. C'est en effet à partir de l'existence humaine comprise comme le «primairement historique $»^{39}$ que l'histoire tant comme science que comme événement sera pensée dans l'ouvrage de 1927. C'est d'ailleurs, selon Paul Ricœur, l'une des avancées fondamentales de la réflexion heideggérienne sur l'histoire que d'avoir pensé celle-ci non pas comme un phénomène public mais à partir de l'individu, à partir du Dasein qui seul donne un sens historique aux choses qui l'entourent ${ }^{40}$. De cette façon, Heidegger s'éloigne des considérations sur l'histoire universelle pour repenser l'histoire à partir de la finitude temporelle propre à l'existence humaine, pierre de touche de toute considération historique.

La reconduction des considérations sur l'histoire universelle à l'historicité facticielle - et donc individuelle - du Dasein comporte son lot de difficultés, mais elle a l'avantage de nous permettre d'inscrire au sein même de l'ontologie fondamentale la problématique de la "réalité historique» (geschichtliche Wirklichkeit). Le mode d'être de l'étant historique - tout comme c'est le cas pour l'étant vohanden ou zuhanden - doit être saisi à partir du Dasein et des rapports qu'il établit avec les étants qui l'entourent. C'est donc dans la compréhension de l'être propre au Dasein que se trouvent les éléments principaux $\mathrm{d}^{\prime}$ une solution aux problèmes d'une ontologie de l'histoire.

Or, lorsque Heidegger pose à nouveau la question de la possibilité d'une telle ontologie dans le cours du semestre d'été 1927, il ajoute d'intéressantes informations. L'étant historique (das geschichtliche

\footnotetext{
${ }^{39} \mathrm{SZ}$, p. 381.

40 P. Ricœur, Temps et récit, tome III : Le temps raconté, Paris, Seuil, 1985, p. 110.
} 
Heidegger et le problème de l'accès phénoménologique à la réalité...

Seiende), écrit alors Heidegger, est un étant que l'on doit caractériser à partir de son appartenance nécessaire au monde. Contrairement à l'étant naturel qui n'est intramondain que de façon contingente, l'étant historique ou culturel n'est tel que grâce à son appartenance à un monde ouvert par un Dasein lui-même historique :

La culture n'est pas au même sens que la nature. Mais nous disons pourtant que les œuvres de la culture, fût-ce l'outil le plus rudimentaire, une fois présentes comme intramondaines, sont en mesure de subsister même en l'absence de tout Dasein historique existant. Il y a ici une corrélation qui mériterait qu'on s'y arrête. Nous ne pouvons l'indiquer que brièvement: tout ce qui est historique au sens du mondo-historial (das Weltgeschichtliche) - les œuvres de la culture dépend de conditions ontologiques toutes différentes suivant qu'il s'agit de leur genèse ou de leur déclin et de leur disparition. Ce sont là des relations qui relèvent de l'ontologie de l'histoire ${ }^{41}$.

Comme nous le voyons, le projet d'une « ontologie de l'histoire » est encore présent à l'esprit de Heidegger bien qu'il renvoie à plus tard une considération en profondeur de ses problématiques. Une étude détaillée des cours suivants - principalement celui de l'hiver 1928-1929 intitulé Einleitung in die Philosophie qui, après avoir traité de la relation entre philosophie et science et philosophie et vision du monde aurait dû se consacrer à la relation entre philosophie et histoire - nous permettrait de montrer que cette problématique finit par s'éclipser au profit d'une réflexion radicalement différente sur l'histoire qui mènera au projet de l'histoire de l'être ${ }^{42}$.

${ }^{41}$ GA 24, 241.

${ }^{42}$ Ce travail a reçu l'appui du Ministerio de Economía y Competitividad d'Espagne (FFI2015-63794P). 